\section{Case Reports in Ophthalmology}

\title{
Optical Coherence Tomography Angiography of Iris Nevus: A Case Report
}

\author{
Davide Allegrini $^{a} \quad$ Giovanni Montesano $^{b} \quad$ Alfredo Pece $^{a}$ \\ ${ }^{a}$ Department of Ophthalmology, Melegnano Hospital, Milan, Italy; ${ }^{b}$ Eye Clinic, San Paolo \\ Hospital, University of Milan, Milan, Italy
}

\section{Keywords}

Iris optical coherence tomography angiography $\cdot$ Iris nevus vasculature $\cdot$ Iris fluorescein angiography

\begin{abstract}
Iris nevus is common: $6 \%$ of patients with suspected iris melanoma have lesions other than melanoma, and $36 \%$ of them are nevi. Iris nevus turns into melanoma in approximately $8 \%$ of cases at a mean of 15 years. This case report provides the first description of an iris tumor examined with iris optical coherence tomography angiography (OCTA) compared to iris fluorescein angiography (IFA). A 60-year-old man with a diagnosis of iris nevus in the left eye was referred to our department for IFA and iris OCTA. The iris vasculature in IFA was visible only in the early phases, but not clearly. OCTA, however, gave visualization of the vascular network and very precisely defined the vessels of the whole lesion, except for the pupillary portion, which was masked by superficial pigment accumulations. IFA and iris OCTA can add information about the vascular architecture compared to slit-lamp biomicroscopy, ultrasound biomicroscopy, and anterior-segment OCT. However, IFA is time-consuming and invasive and can - very occasionally - cause serious adverse reactions. In contrast, OCTA defines the texture of the iris vasculature better. In conclusion, OCTA is a new method, easy to execute,
\end{abstract}


Case Reports in
Ophthalmology

Case Rep Ophthalmol 2016;7:172-178

DOI: $10.1159 / 000450572$

C 2016 The Author(s). Published by S. Karger AG, Basel www.karger.com/cop

Allegrini et al:: Optical Coherence Tomography Angiography of Iris Nevus: A Case Report

needing no dye injection, and provides useful information on the vascular network of iris lesions. It could therefore be helpful in the diagnosis and follow-up of these lesions.

(C) 2016 The Author(s)

Published by S. Karger AG, Basel

\section{Introduction}

Iris nevus is common: a clinical study suggested that these nevi are present in $9 \%$ of a general population [1]. A recent investigation on 3,610 patients with iris tumors found that iris nevus was the most common condition including $25 \%$ of all iris lesions in children, $36 \%$ in young adults, and $47 \%$ in middle-aged and senior adults [2]. Several lesions of the iris may look clinically like melanoma. Shields et al. [3] studied 200 patients referred for suspected iris melanoma, and 158 (76\%) were found to have lesions other than melanoma; these are termed pseudomelanomas. The most common pseudomelanomas included iris cyst (38\%) and iris nevus (31\%). Iris nevus turns into melanoma in approximately $8 \%$ of cases at an average of 15 years [4].

Iris fluorescein angiography (IFA) can be helpful in establishing the malignancy of iris tumors and in their diagnosis and follow-up. Unlike for ultrasound biomicroscopy (UBM) and anterior-segment optical coherence tomography (ASOCT) [5], there are no recent studies on the utility of IFA in the evaluation of iris nevi. In the past, studies on IFA have given conflicting results about its effective diagnostic role for this type of lesion. However, the recent development of OCT angiography (OCTA) allows visualization of the capillary retinal layers for the construction of microvascular flow maps. This procedure, which does not need sodium fluorescein dye injection, can also be used to study iris vessels. This case report offers the first description of an iris tumor vasculature examined with OCTA compared to IFA.

\section{Case Report}

A 60-year-old man with suspected iris nevus in the left eye was referred to our department for IFA and iris OCTA. The patient was being followed at another center, which had already performed (1) a complete ophthalmic evaluation [anterior segment (AS), intraocular pressure, and fundus oculi]; (2) a photography of the AS; (3) UBM, and (4) ASOCT. The diagnosis was iris nevus. All these examinations had been repeated every 6 months for the first 2 years, then annually. At the last eye check, the lesion had been stable for 10 years.

For IFA, we used the digital fluorescein angiogram instrument (TRC-50DX; Topcon, Itabashi-ku, Tokyo, Japan) with the focus set on +30.00 D. Angiographic images were captured after slow injection of $3 \mathrm{ml}$ of fluorescein dye, and images were taken at $30 \mathrm{~s}$ and $3 \mathrm{~min}$. The OCTA examination was done with the AngioVue system (Optovue Inc., Fremont, Calif., USA). This instrument operates at approximately $840 \mathrm{~nm}$ wavelength and 70,000 Ascans per second to acquire OCTA volumes consisting of 2 repeated B-scans from 304 sequential, uniformly spaced locations. All scans were automatically processed by the internal software to reduce motion artefacts (ReVue V.2014.2.0.15). The examination was done in AngioRetina mode, but with the AS optical adaptor lens and without autofocus, manually adjusting the xyz-axes to bring the iris vessels into focus. OCTA scans were captured in both eyes, always by the same operator. The images were acquired in $3 \times 3$ and $6 \times 6$ cubes.

At our ophthalmological evaluation, the patient presented s best-corrected visual acuity of 20/20 and an intraocular pressure of $16 \mathrm{~mm} \mathrm{Hg}$ in both eyes. The AS photography showed 


\section{Case Reports in Ophthalmology}

a blue iris with a hyperpigmented lesion measuring $2.60 \times 2.26 \mathrm{~mm}$, localized in the inferior nasal sector, with slight deformation of the pupil; the pupillary portion was the most pigmented area (about one third of the entire nevus surface) (fig. 1). IFA indicated early fluorescence in the tumor and a tendency for dye to leak late, progressively covering the nevus vasculature. The largest pigmented area of the pupillary nevus portion caused an irregular hypofluorescent zone in the angiogram, which persisted into the late phases. The vascular network was not clearly observable, and then only at the early time points. It seemed irregular but was not clearly visible in detail (fig. 2).

Iris OCTA gave a better visualization of the nevus vascular network, and in the case described, it was clearly observable on the entire lesion except for the pupillary portion, which was masked by superficial pigment accumulation. The nevus vasculature appeared anarchic, in marked contrast to the linear course of the iris vessels (fig. 3). Segmentation was manually corrected by moving the top and bottom layers to include the whole region of interest. Although this meant that part of the apex of the nevus was not included, no OCTA data were excluded in the projection depicted in figure 3. Iris OCT scans were taken upside down to enhance the signal from deeper layers of the nevus, as is sometimes done in enhanced depth imaging [6].

In our eye check, the features and size of the lesion appeared stable. This is compatible with a diagnosis of iris nevus. However, the patient had not previously been examined by both IFA and OCTA, so we recommended repeating these tests after about 6 months to assess any changes in the leakage and vascular network.

\section{Discussion}

Iris nevus carries a low risk of progression to melanoma. In a recent series of 1,611 eyes with iris nevus, Shields et al. [4] found 6 predictive clinical factors for growth (A-F): AGE young, Blood (hyphema), Clock hour inferior, Diffuse (involving entire iris surface) configuration, Ectropion uveae, and Feathery margins. Tumor basal diameter and thickness were measured in millimeters by slit-lamp biomicroscopy and, when available, UBM and ASOCT [4]. For the A-F parameters, the slit lamp, UBM, and ASOCT are useful in the follow-up to check the growth of the iris nevus. They provide information on the size, infiltration, and localization of iris tumors [5] but do not show the vascular architecture.

It is not easy to clinically establish a reliable diagnosis of pigmented iris tumors; however, the iris lesion vasculature may show alterations characteristic of malignant forms, so this is useful for a correct diagnosis in case of a pigmented neoformation of the iris [7]. IFA can show the vascular network but only with intravenous sodium fluorescein dye; in addition, it is time-consuming and invasive and can - very occasionally - cause serious adverse reactions. These aspects make it unsuitable for periodic checks. In contrast, the iris vascularization can be studied with OCTA without the need for fluorescein.

IFA, unlike OCTA, depicts blood vessel leakage. If early, this can impede visualization of the iris vessels. Although the importance of vascular leakage in iris tumors is debated, there are reports that it may be indicative of malignancy [8], especially if early or profuse [9-12], though others maintain that it is associated with both benign and malignant tumors $[10$, $11,13]$.

Both IFA and iris OCTA depict the vascular network of the neoformation and the effects of its growth on surrounding tissues. These features are very important, as in fact some authors believe that a geometric and organized vascular picture may be associated with benign 
Case Reports in
Ophthalmology

Case Rep Ophthalmol 2016;7:172-178

DOI: $10.1159 / 000450572$

C 2016 The Author(s). Published by S. Karger AG, Basel www.karger.com/cop

Allegrini et al:: Optical Coherence Tomography Angiography of Iris Nevus: A Case Report

lesions [11, 12], and others maintain that a disorganized, irregular tumor vasculature can be a sign of malignancy $[9,11,13]$. However, in our patient, the nevus vascularization, although partially masked by pigment in the pupillary portion, was more clearly visible with OCTA than with IFA. The vascular nevus network was not clearly visible with IFA because it was masked by rapid loss of dye from the vessels. Instead, with OCTA, it could be seen better on the entire lesion - except for the part covered by superficial pigment - because, as no dye is used, there is no interference due to early leakage (fig. 2, 3).

In conclusion, we believe that OCTA examination of the iris vasculature may be useful both for the diagnosis and follow-up to evaluate the tumor edges and to observe the vascular structure of the tumor without dye injection. Further studies and experience will be necessary to confirm this preliminary report.

\section{Conclusion}

The case reported provides the first OCTA description of an iris nevus. This instrument has already been employed to study the retina, and we reprogrammed it for the study of iris lesions. The prognostic value of vascular leakage in iris tumors is not clear, but details of the vascular network could provide additional information in relation to the A-F criteria regarding malignancy or benignity of a pigmented iris tumor. Therefore, iris OCTA, offering ease of execution and safety, could be helpful in the follow-up of iris lesions together with slit-lamp biomicroscopy, UBM, and ASOCT to check on the progression of pigmented iris tumors.

\section{Acknowledgment}

This study was supported by the Retina 3000 Foundation.

\section{Authors' Contribution}

All authors contributed to the conception and design, data acquisition, and data interpretation; all authors drafted the article and approved its final version.

\section{Funding Sources}

This study received no specific grant from any funding agency in the public, commercial, or not-for-profit sectors.

\section{Statement of Ethics}

The patient signed a comprehensive consent form according to Good Clinical Practice guidelines before proceeding with all examinations and treatments. It satisfied all the requirements of the Declaration of Helsinki and Italian national law for the protection of personal data. The local ethics committee decided that no formal ethics approval was required in this particular case. 


\section{Disclosure Statement}

The authors have no interests in any instrument/product mentioned in the article.

\section{References}

1 Seregard S, af Trampe E, Månsson-Brahme E, Kock E, Bergenmar M, Ringborg U: Prevalence of primary acquired melanosis and nevi of the conjunctiva and uvea in the dysplastic nevus syndrome. A casecontrol study. Ophthalmology 1995;102:1524-1529.

2 Shields CL, Kancherla S, Patel J, et al: Clinical survey of 3680 iris tumors based on age at presentation. Ophthalmology 2012;119:407-414.

-3 Shields JA, Sanborn GE, Augsburger JJ: The differential diagnosis of malignant melanoma of the iris. A clinical study of 200 patients. Ophthalmology 1983;90:716-720.

-4 Shields CL, Kaliki S, Hutchinson A, Nickerson S, Patel J, Kancherla S, Peshtani A, Nakhoda S, Kocher K, Kolbus E, Jacobs E, Garoon R, Walker B, Rogers B, Shields JA: Iris nevus growth into melanoma: analysis of 1611 consecutive eyes: the ABCDEF guide. Ophthalmology 2013;120:766-772.

-5 Bianciotto C, Shields CL, Guzman JM, Romanelli-Gobbi M, Mazzuca D Jr, Green WR, Shields JA: Assessment of anterior segment tumors with ultrasound biomicroscopy versus anterior segment optical coherence tomography in 200 cases. Ophthalmology 2011;118:1297-1302.

-6 Spaide RF, Koizumi H, Pozzoni MC: Enhanced depth imaging spectral-domain optical coherence tomography. Am J Ophthalmol 2008;146:496-500.

7 Brancato R, Bandello F, Lattanzio R: Atlas of Iris Fluorescein Angiography, ed 1. Milano, Ghedini Editore, 1995.

-8 Cheng H, Bron A, Easty D: A study of iris masses by fluorescein angiography. Trans Ophthalmol Soc UK 1971;91:199-205.

-9 Dart JK, Marsh RJ, Garner A, Cooling RJ: Fluorescein angiography of anterior uveal melanocytic tumours. Br J Ophthalmol 1988;72:326-337.

10 Demeler U: Fluorescence angiographical studies in the diagnosis and follow-up of tumours of the iris and ciliary body. Adv Ophthalmol 1981;42:1-17.

11 Jakobiec FA, Depot MJ, Henkind P, Spencer WH: Fluorescein angiographic patterns of iris melanocytic tumours. Arch Ophthalmol 1982;100:1288-1299.

12 Brovkina AF, Chichua AG: Value of fluorescein iridography in diagnosis of tumours of the iridociliary zone. Br J Ophthalmol 1979;63:157-160.

$\$ 13$ Kottow M: Fluorescein angiographic behaviour of iris masses. Ophthalmologica (Basel) 1977;174:217223. 


\begin{tabular}{ll} 
Case Reports in & \multicolumn{3}{c}{\begin{tabular}{l} 
Case Rep Ophthalmol 2016;7:172-178 \\
\cline { 2 - 3 } DOI: 10.1159/000450572
\end{tabular}} & $\begin{array}{l}\text { @ } 2016 \text { The Author(s). Published by S. Karger AG, Basel } \\
\text { www.karger.com/cop }\end{array}$ \\
\hline Allegrini et al.: Optical Coherence Tomography Angiography of Iris Nevus: A Case Report
\end{tabular}

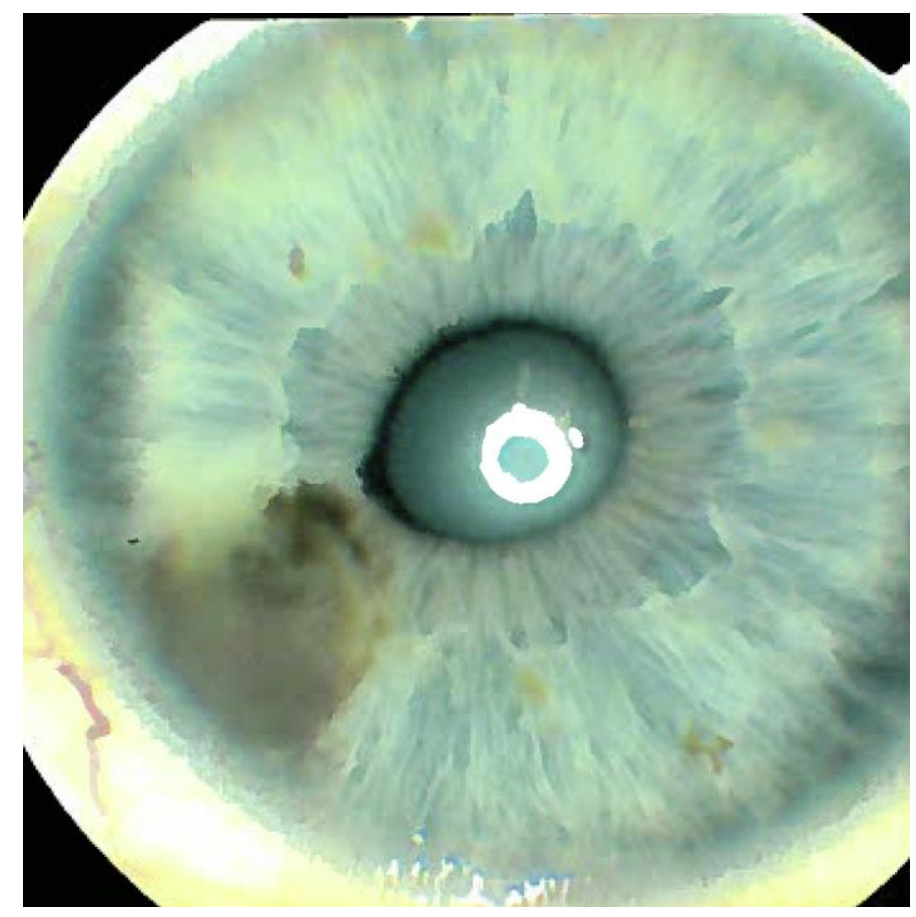

Fig. 1. Photography of the AS of the left eye.

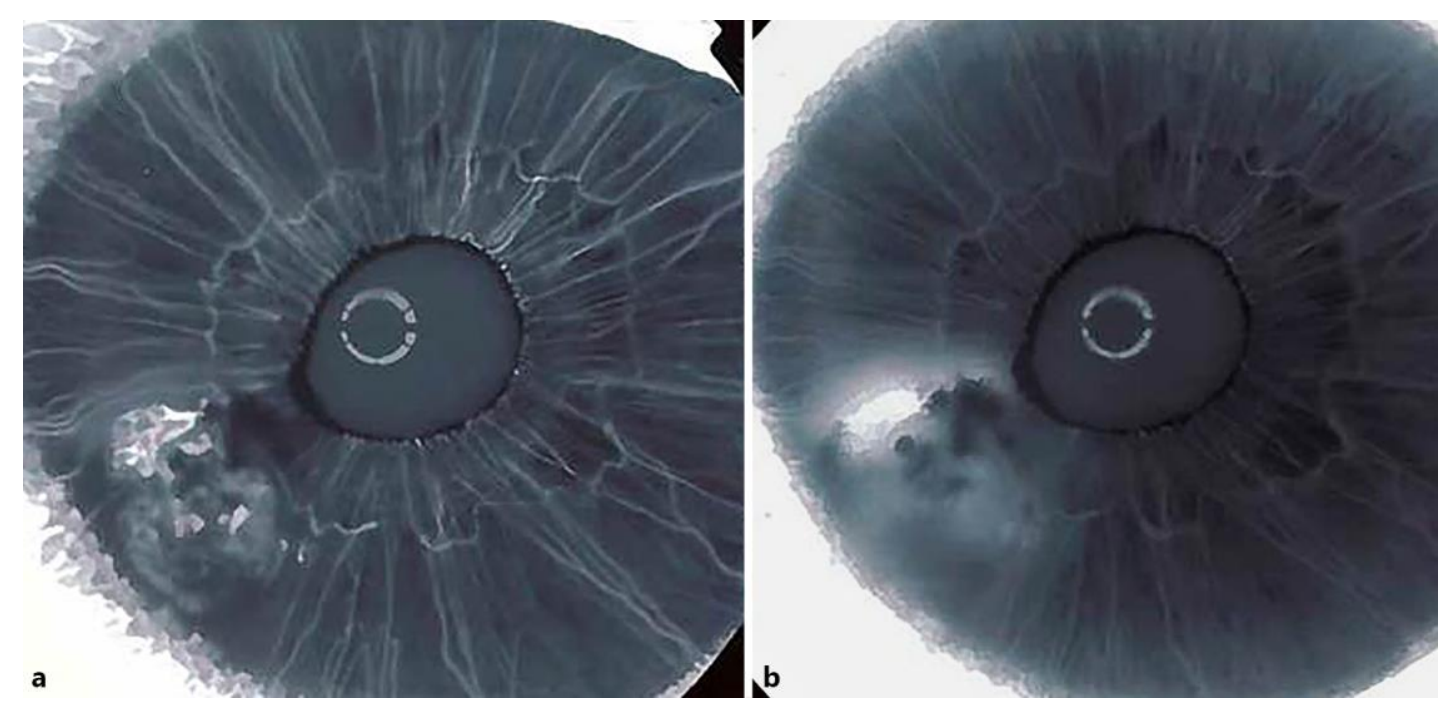

Fig. 2. IFA of the left eye: $30 \mathrm{~s} \mathrm{(a)} \mathrm{and} 3 \mathrm{~min}(\mathrm{~b})$ after injection of fluorescein dye. 


\begin{tabular}{|c|c|}
\hline \multirow{2}{*}{\multicolumn{2}{|c|}{ Case Rep Ophthalmol 2016;7:172-178 }} \\
\hline & \\
\hline DOI: $10.1159 / 000450572$ & $\begin{array}{l}\text { (c) } 2016 \text { The Author(s). Published by S. Karger AG, Basel } \\
\text { www.karger.com/cop }\end{array}$ \\
\hline
\end{tabular}

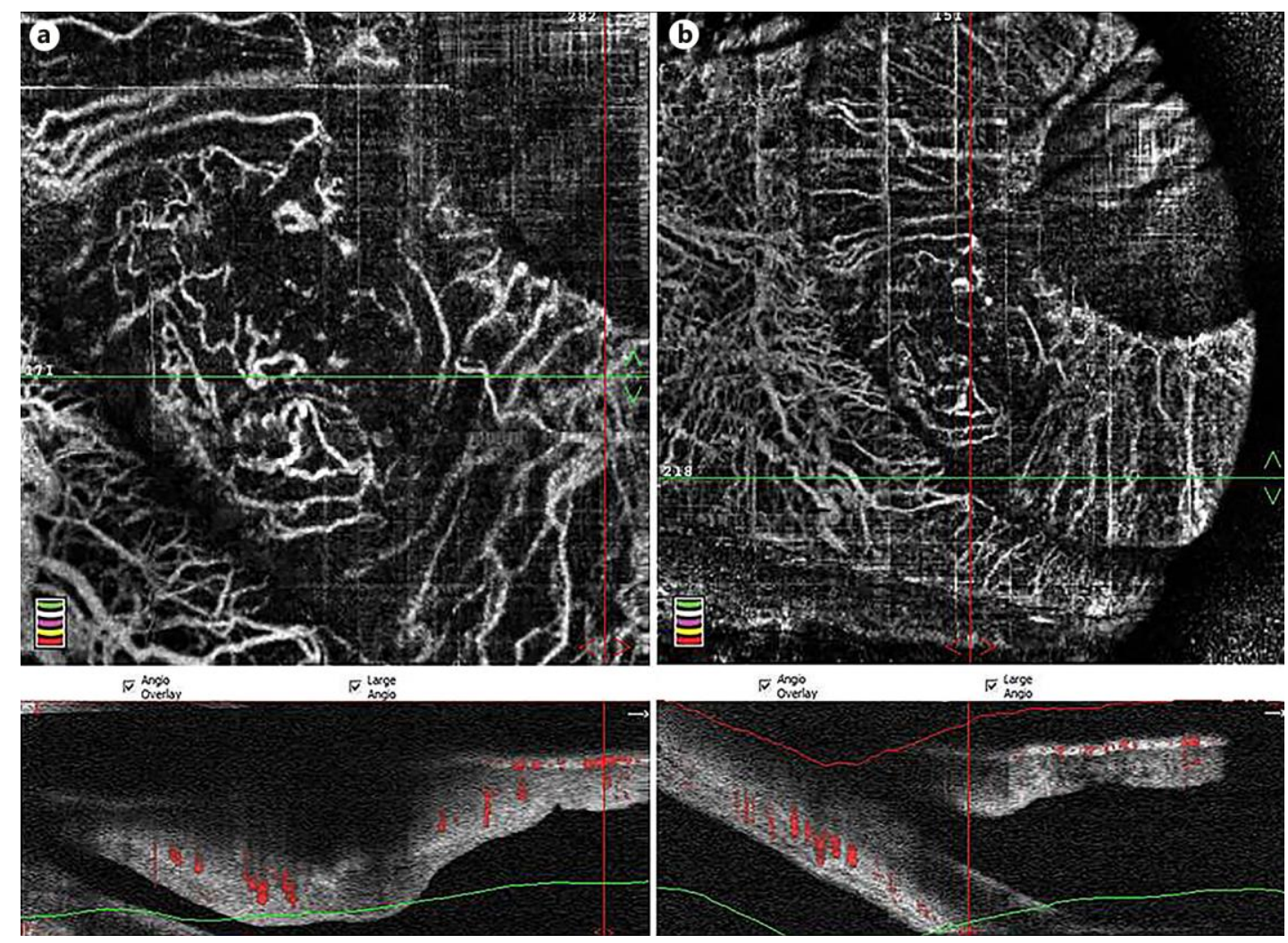

Fig. 3. Iris OCT and OCTA of the left eye: $3 \times 3$ (a) and $6 \times 6$ (b) cubes. 